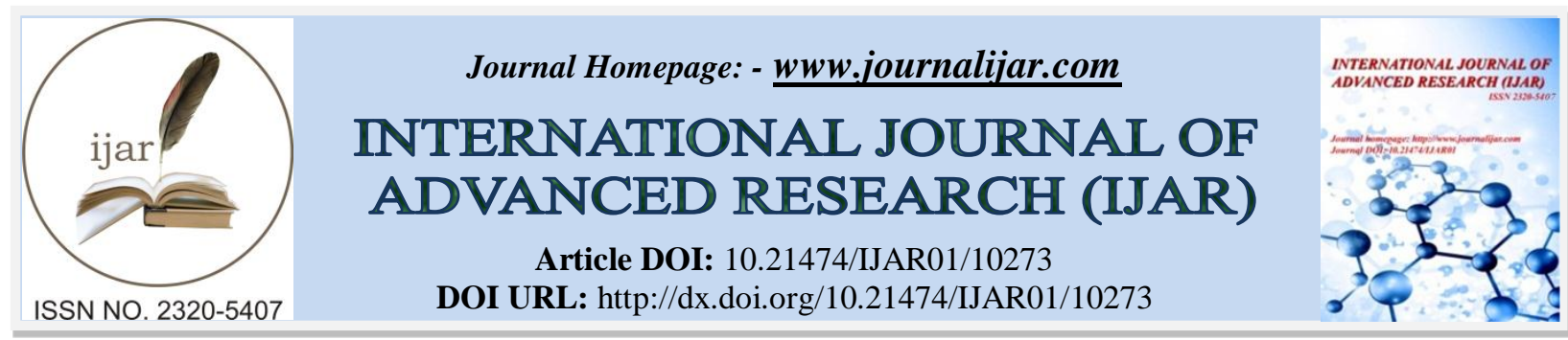

RESEARCH ARTICLE

\title{
PREVALENCE AND RISK FACTORS OF POLYCYSTIC OVARY DISEASE IN KINGDOM OF SAUDI ARABIA
}

Dr. Afaf Abdulrahman Yaslam ${ }^{1}$, Hoda Jehad Abousada ${ }^{2}$, Dr. Nejood Mosab Waggas ${ }^{2}$, Dr. Haifa Saeed AlMufarrih $^{2}$, Dr. Amal Bakheet Alzahrani' ${ }^{2}$, Dr. Razan Anwar Ahmad Kurdi' ${ }^{2}$ Dr. Aliyah Hijazi Alsayed Ahmed Hijazi ${ }^{2}$, Dr. Naif Ali Alayfan ${ }^{2}$ and Dr. Saad Saud Alotaibi ${ }^{2}$

1. Consultanat OB/GYN, Obstetric And Gynecology Department, King Abdullah Medical Complex, Jeddah, Saudi Arabia.

2. Medical Intern, Ibn Sina National College, KSA (Postal Address: jeddah, Al-Thaghr Neighborhood., 22338.

\section{Manuscript Info}

Manuscript History

Received: 20 November 2019

Final Accepted: 23 December 2019

Published: January 2020

\section{Abstract}

Copy Right, IJAR, 2020,. All rights reserved.

\section{Introduction:-}

Polycystic ovary syndrome (PCOS) is a hormonal disorder common among women of reproductive age. Women with PCOS may have infrequent or prolonged menstrual periods or excess male hormone (androgen) levels. The ovaries may develop numerous small collections of fluid (follicles) and fail to regularly release eggs.

The exact cause of PCOS is unknown. Early diagnosis and treatment along with weight loss may reduce the risk of long-term complications such as type 2 diabetes and heart disease. (Mayo Clinic, 2019)

\section{Rationale:-}

The importance of this research is that it is concerned with the health of women in the reproductive period, polycystic ovary syndrome disease may be one of the important reasons for the infertility, which threatens the happiness of women in her life, motherhood is an irreplaceable feeling and an indescribable feeling. Polycystic ovary syndrome, this may contribute to the treatment of the root cause and prevent the occurrence and control early.

\section{Literature review:-}

According to the other previous study like:

1. Risk factors associated with metabolic syndrome and cardiovascular disease among women with polycystic ovary syndrome in Tabuk, Saudi Arabia, the conclusion was: PCOS is associated with the risk of development of MS, and CVD. Screening for early detection of PCOS and MS and the application of cohort studies are recommended to better explore the role of PCOS in the development of CVD and to assess the significance of interventions. (Shaman, Mukhtar and Mirghani, 2017), Which means that this is quite consistent with our existing research theory as we have been keen to mention the relationship between the PCO and Ms, otherwise we cannot forget the relationship between these and diabetes, which has long been called the chronic and deadly friendly disease.

2. Body weight reduction and metformin, Roles in polycystic ovary syndrome.

\section{Corresponding Author:- Hoda Jehad Abousada}

Address:- Medical Intern, Ibn Sina National College, KSA (Postal Address: jeddah, Al-Thaghr Neighborhood., 22338. 


\section{The result was:-}

PCOS women had significantly higher values than the healthy women in most of the measurements. Metformin and weight reduction therapy resulted in a significant decrease in the fasting insulin, glucose/insulin ratio and HOMAIR. Metformin and weight reduction therapy resulted in a significant decrease in the lipid parameters, testosterone, LH/FSH ratio, SHBG, and prolactin levels. HOMA-IR was significantly higher in women with PCOS. HOMA-IR was positively correlated with testosterone, estradiol, TG, total cholesterol and LDL-cholesterol parameters, and negatively correlated with HDL-cholesterol and FSH levels. (Al-Nozha O, 2013) I fully agree with this wonderful and useful research as it touched on the subject of weight gain and its relationship with polycystic ovary syndrome with other diseases, it is now even associated with PCOS, as doctors have become their first advice for treating PCOS is low weight. Many women believe that being overweight only hurts her body shape, but the subject is much higher.

\section{Hirsutism in Saudi females of reproductive age: a hospital-based study:}

The result was: Polycystic ovary syndrome (PCOS) was the cause of hirsutism in 83 patients (82\%) followed by idiopathic hirsutism (IH) in 11 patients (11\%). (Al-Ruhaily, Malabu and Malabu, 2019)

Sometimes we associate things from an angle where we can see better, as hirsutism is a risk factor for the polycystic ovary syndrome disease. The results in this research indicate the strength of this factor.

\section{Research question:}

Questionnaire.

\section{Aim:}

To determine the prevalence and risk factors of PCO.

\section{The sub- objectives:}

1. Determine the age group most affected by this disease.

2. Determine the relationship between late puberty and risk of PCO disease.

3. Determine the relationship between obesity and increased risk of PCO disease.

4. Identify the most common risk factors for this disease.

5. Determine the relationship of diabetes and PCO disease.

6. Effect of PCO disease on infertility.

\section{Method and Design:-}

\section{Study design:}

Cross sectional study.

\section{Study area:}

This study will be conducted in kingdom of saudia Arabia.

\section{Study setting:}

The study will be carried out by questionnaire.

\section{Study population:}

Adult females in reproductive age IP and OP.

\section{Inclusion criteria:}

females

\section{Exclusion criteria:}

Males 


\section{Data Collection Methods:-}

Data collection tool:

Self-administered questionnaire partially constructed by the researcher with reference to already made questionnaire in another study .Validity will be checked by at consultant.

\section{Data collection technique:}

The researcher will distribute the questionnaire.

Study sample and technique:

Sample size:

1718

\section{Sample technique:}

Questionnaire.

\section{Data entry and statistical analysis:}

The data will be entered into a personal computer and it

will be analyzed using Statistical Package for the Social Sciences (SPSS).

\section{Results:-}

Questionnaire Prevalence and risk factors of PCO in kingdom of Saudi Arabia. 1718 responses were collected showing the following

\section{Results And Conclusion:-}

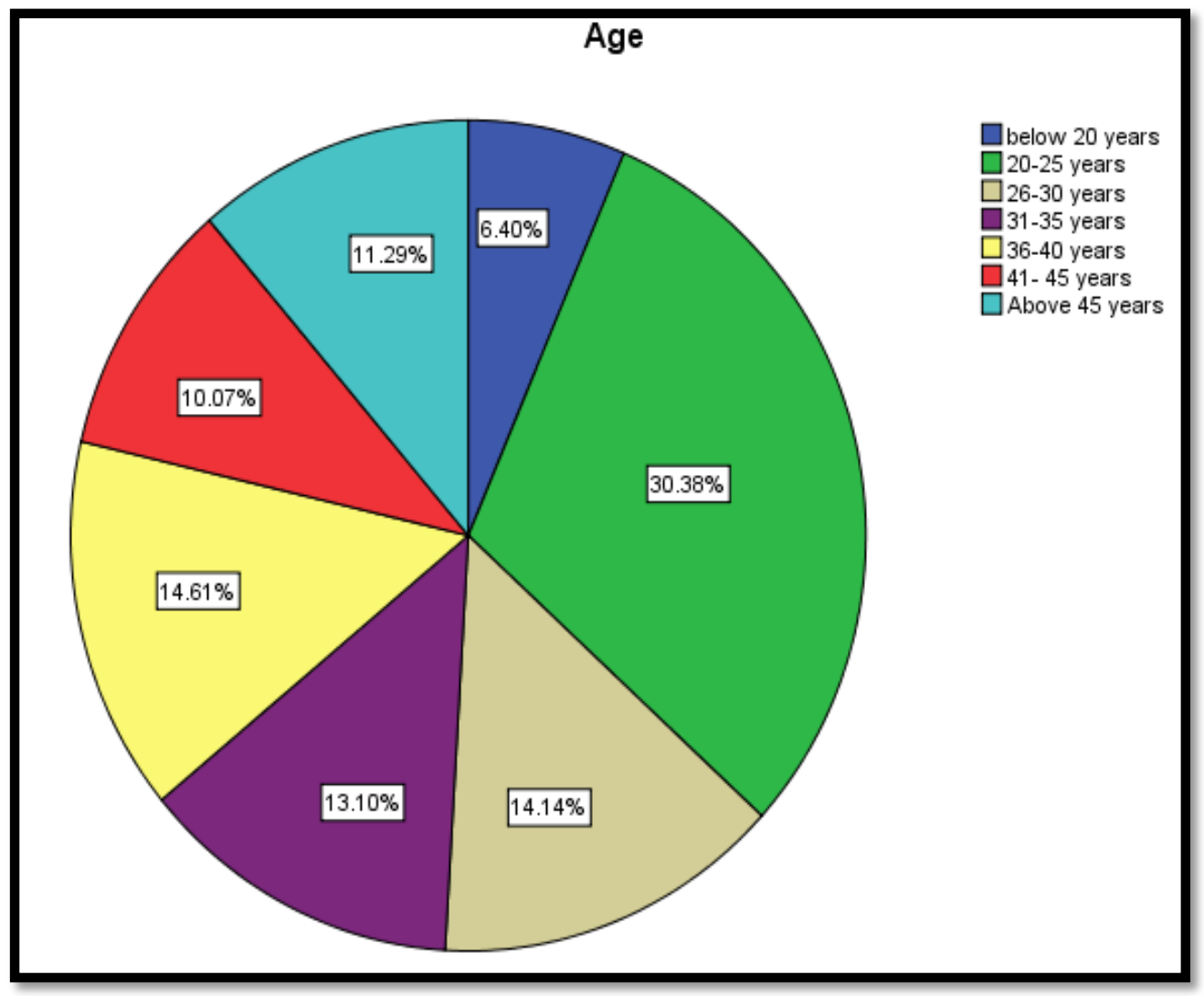

The results indicate that most of the respondents are between 20-25 years. 


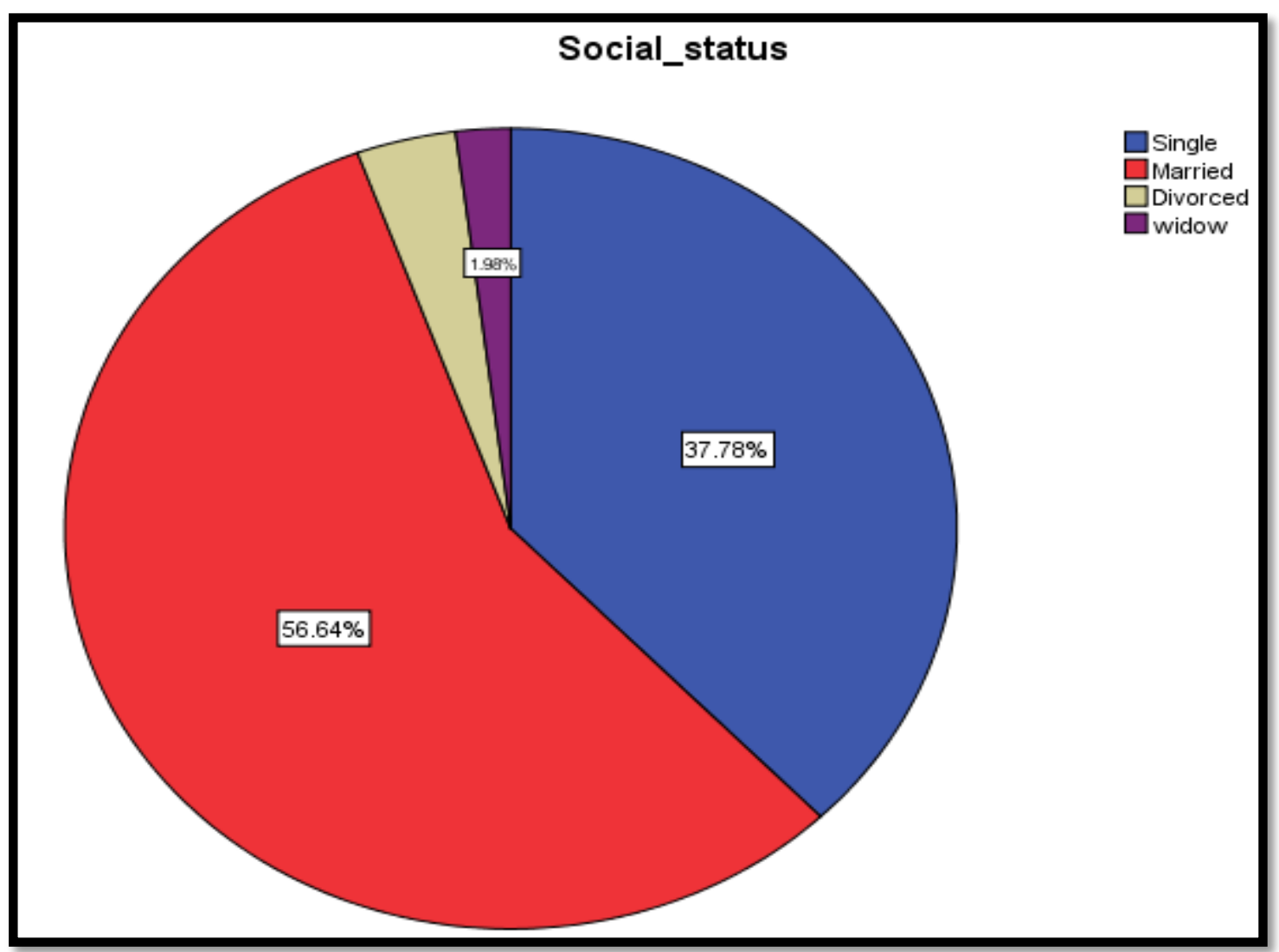

The results indicate that most respondents are married.

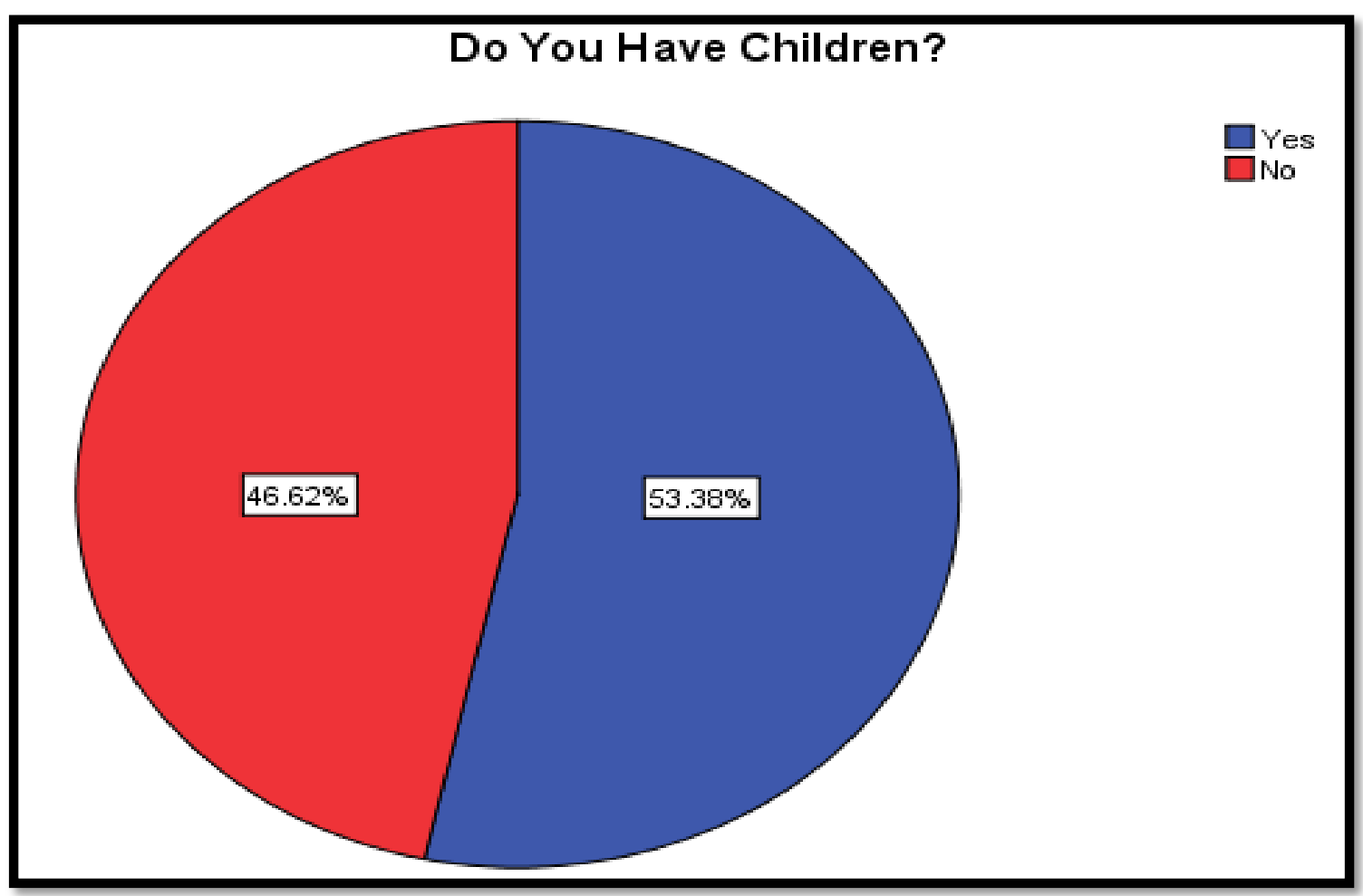


The results show that more than half of the respondents have children.

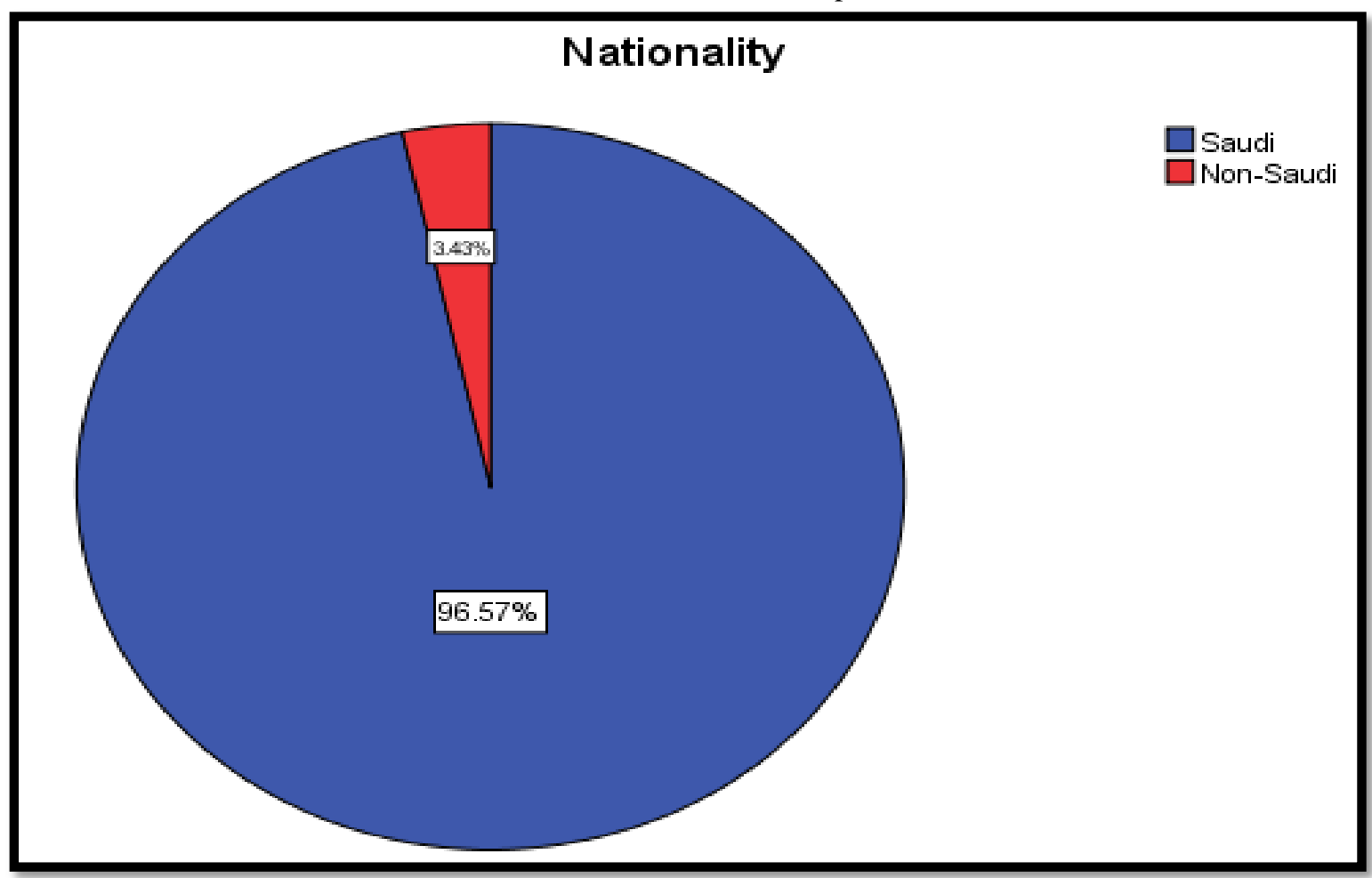

The results indicated that most respondents Saudis.

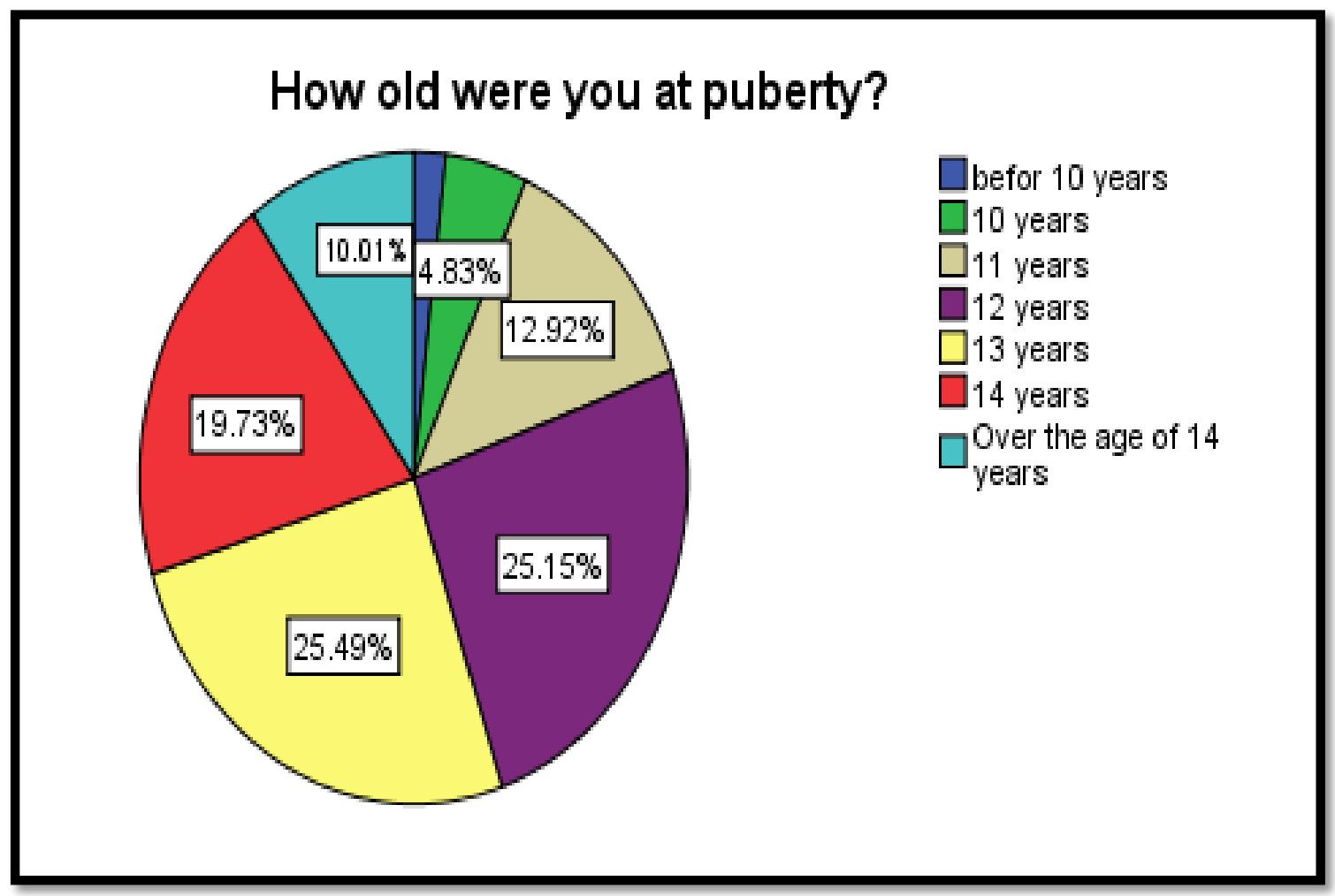

The answer of most respondents to the question of the duration is 12 and 13 years old. 


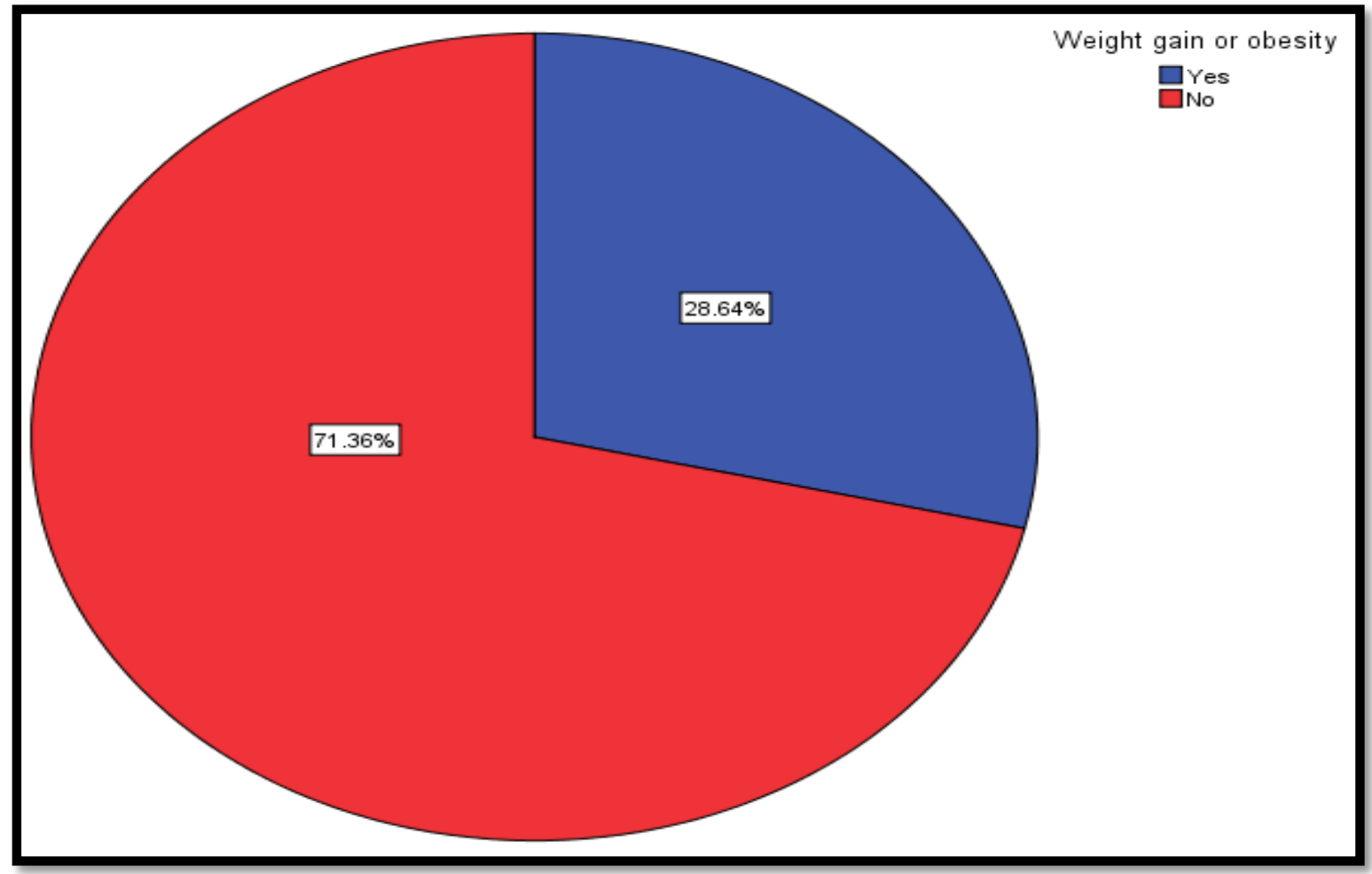

The results indicated that $28.64 \%$ of the respondents suffer from weight gain or obesity.

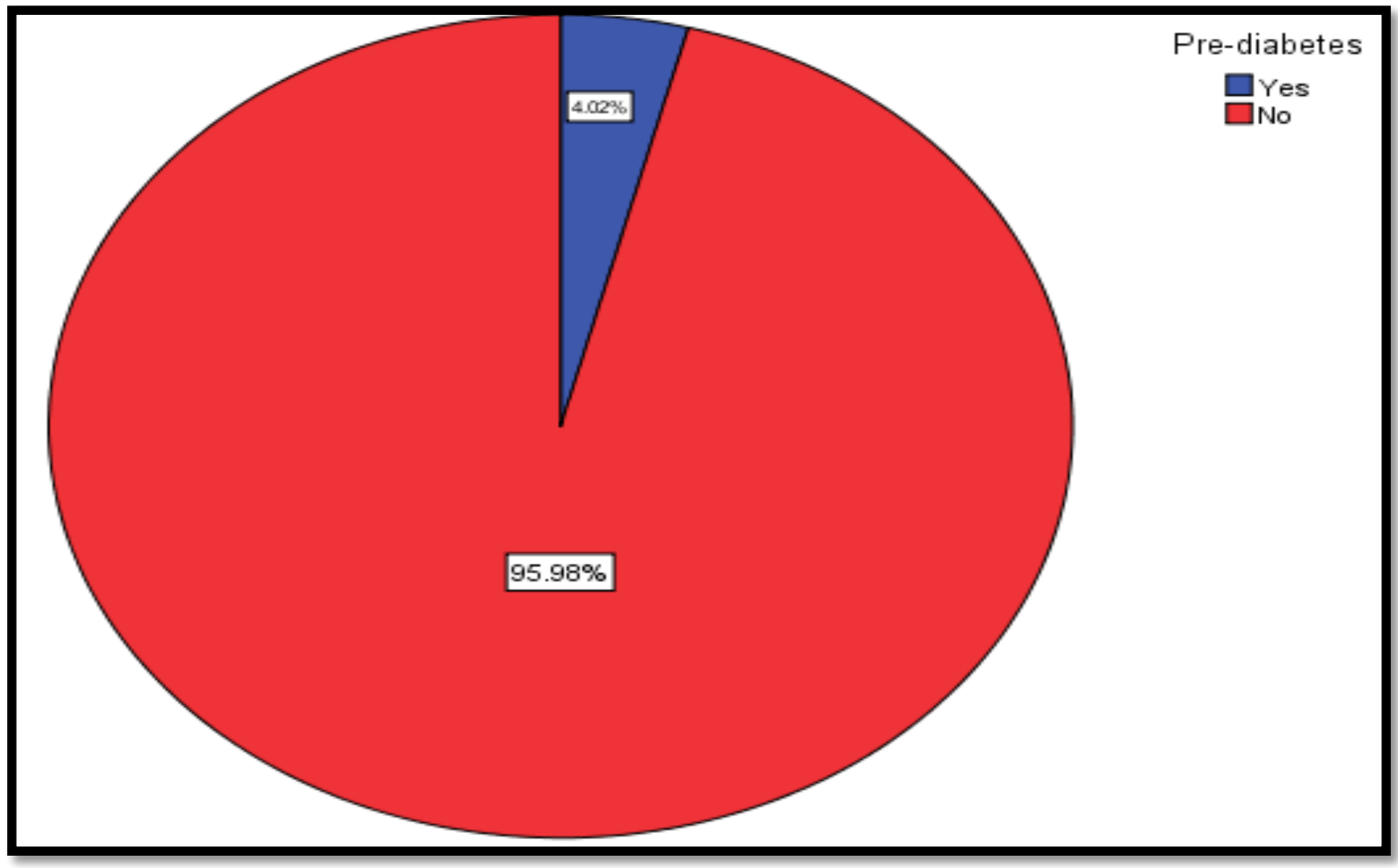

The results indicated that only $4 \%$ of the respondents suffer from pre-diabetes. 


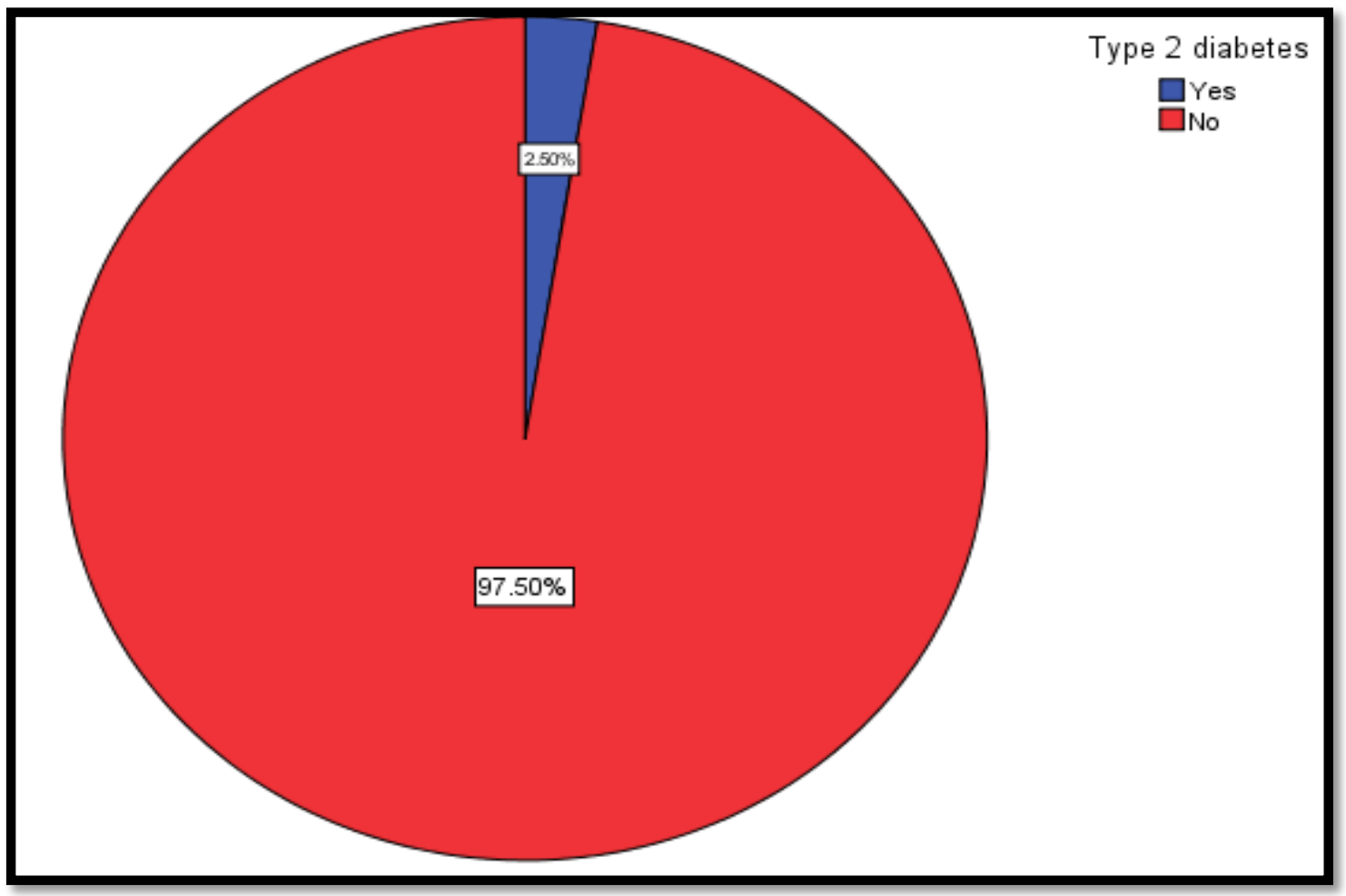

The results indicated that only $2.50 \%$ of the respondents suffer from Type 2 diabetes.

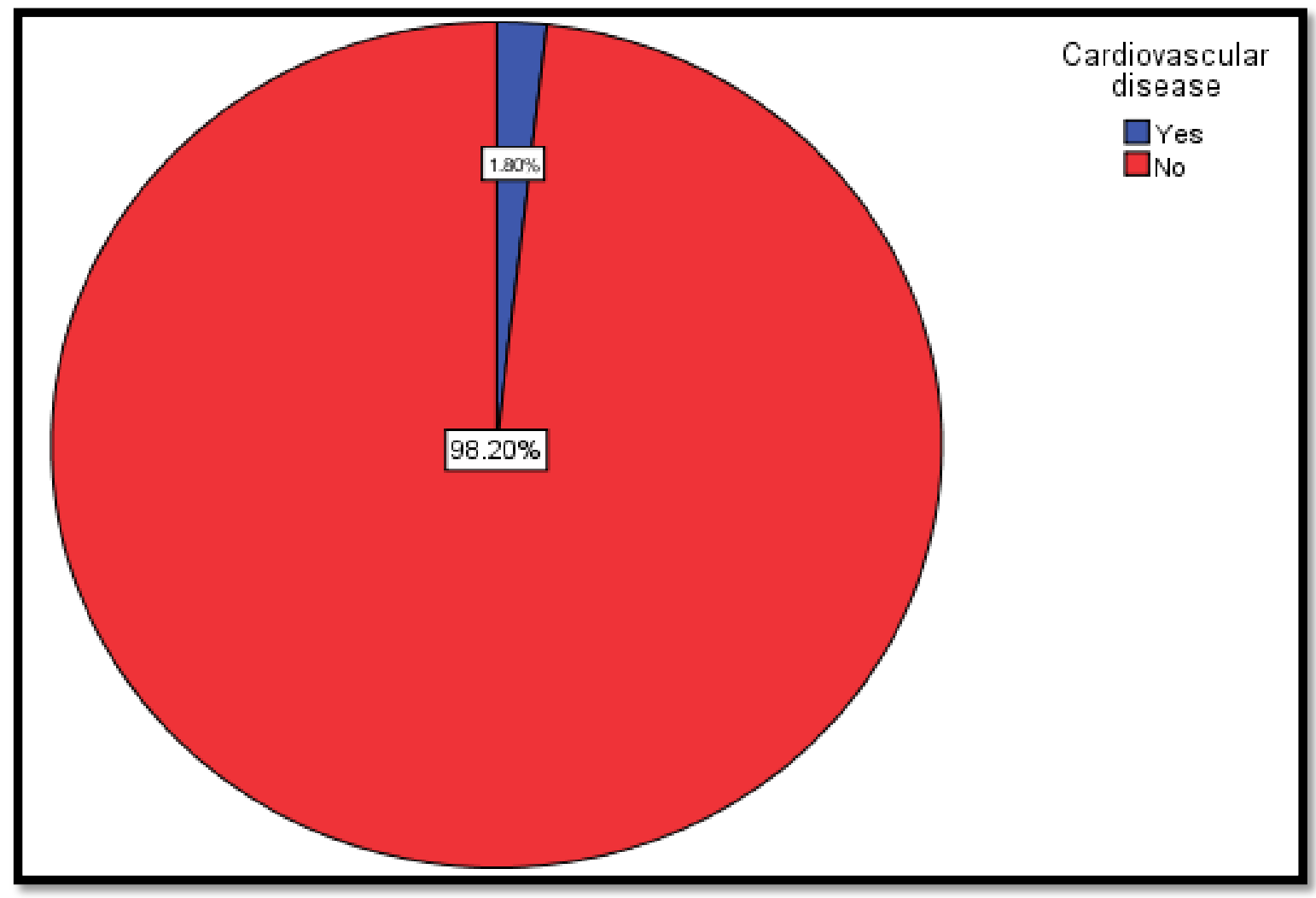

The results indicated that only $1.80 \%$ of the respondents suffer from Cardiovascular disease. 


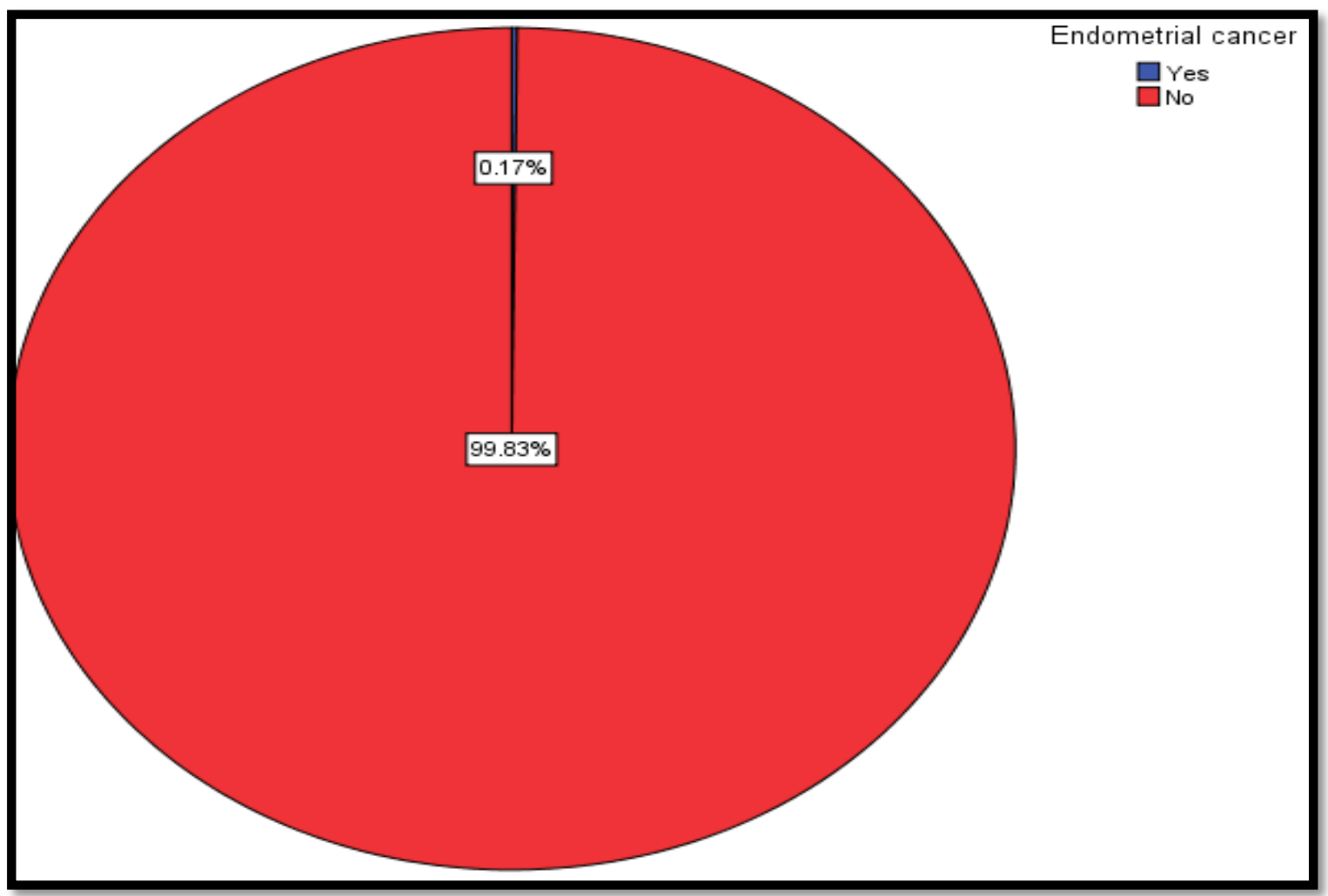

The results indicated that only $0.17 \%$ of the respondents suffer from Endometrial cancer.

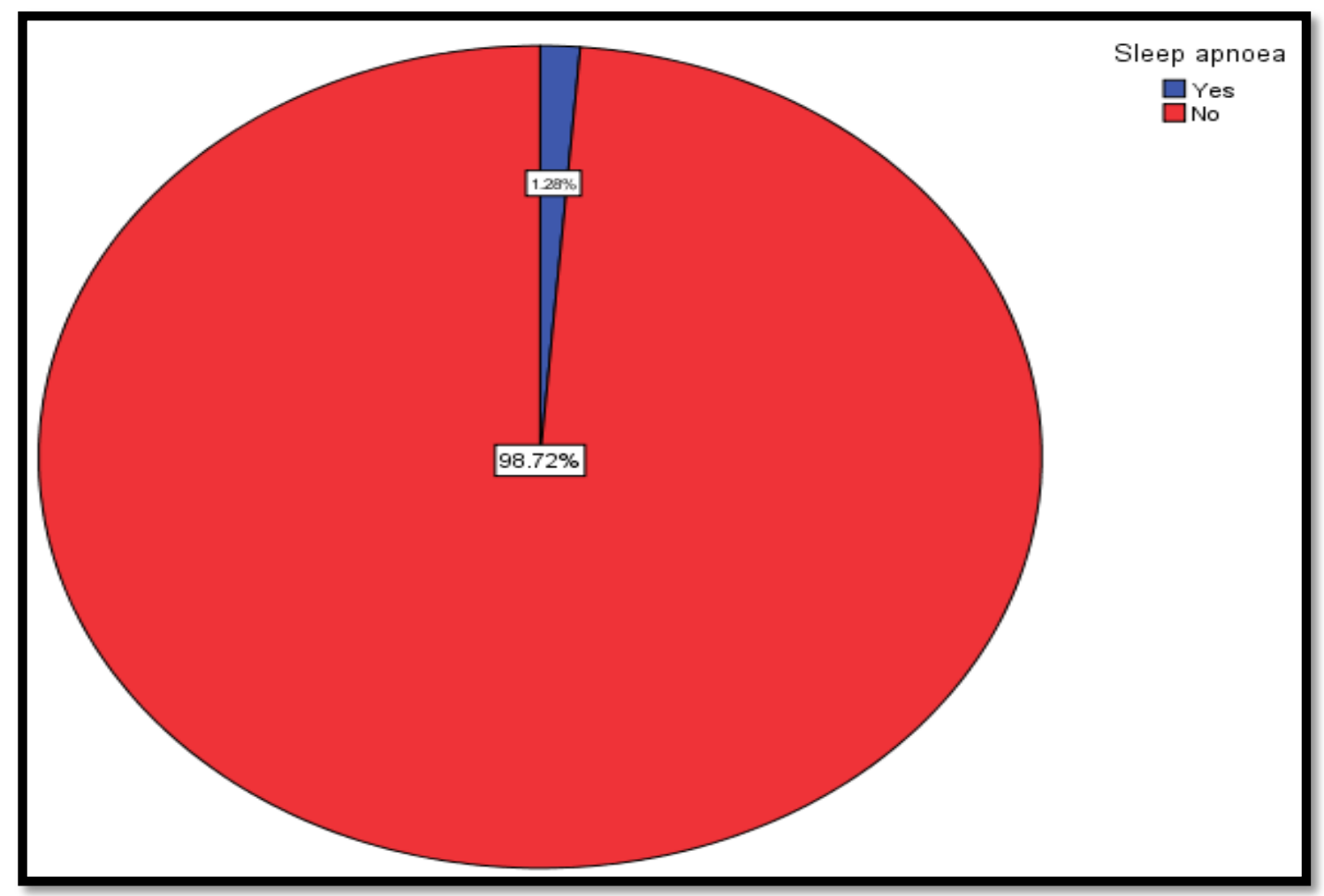

The results indicated that only $1.28 \%$ of the respondents suffer from Sleep apnoea. 


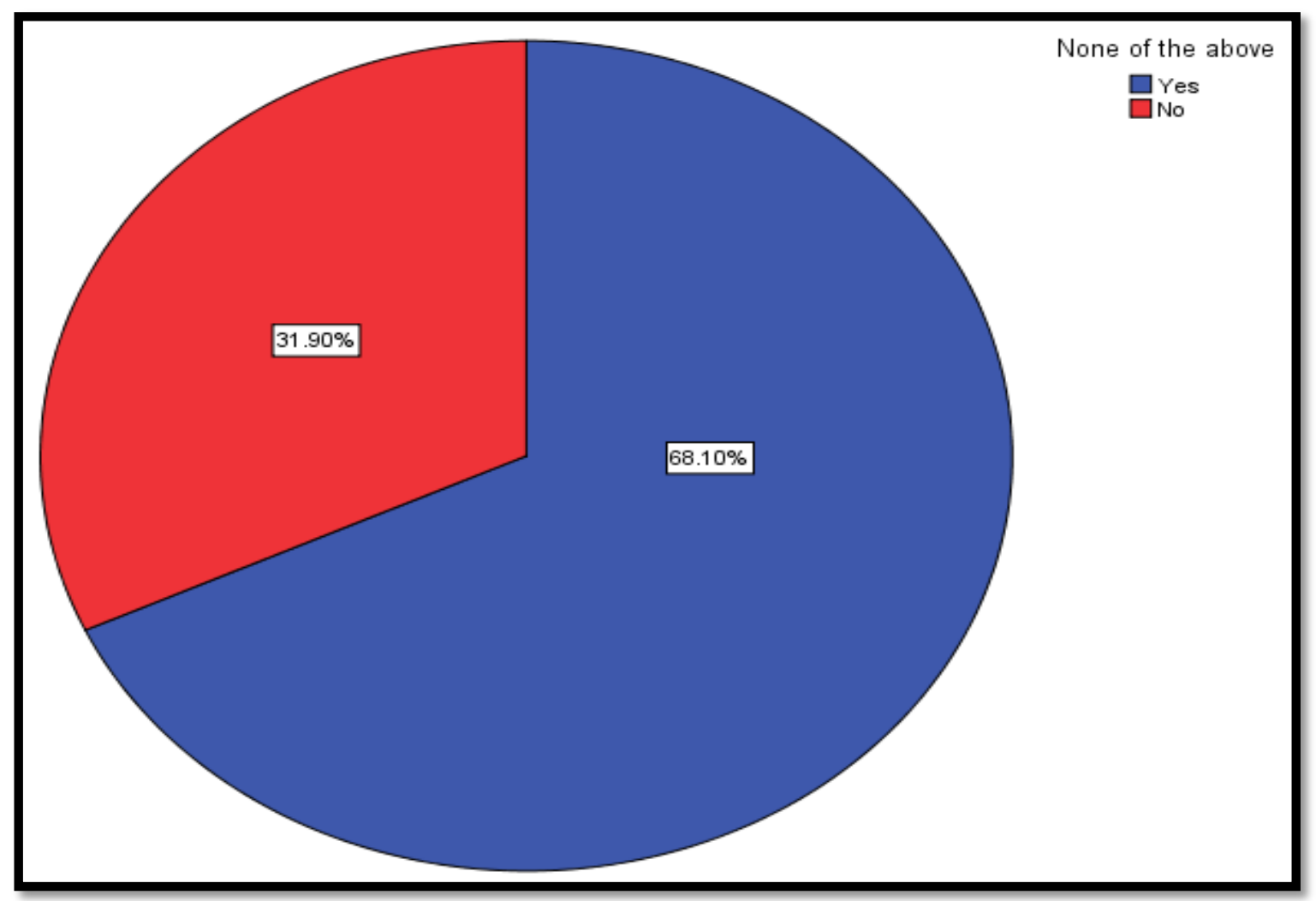

The results indicated that $68.10 \%$ of the respondents do not suffer from the diseases.

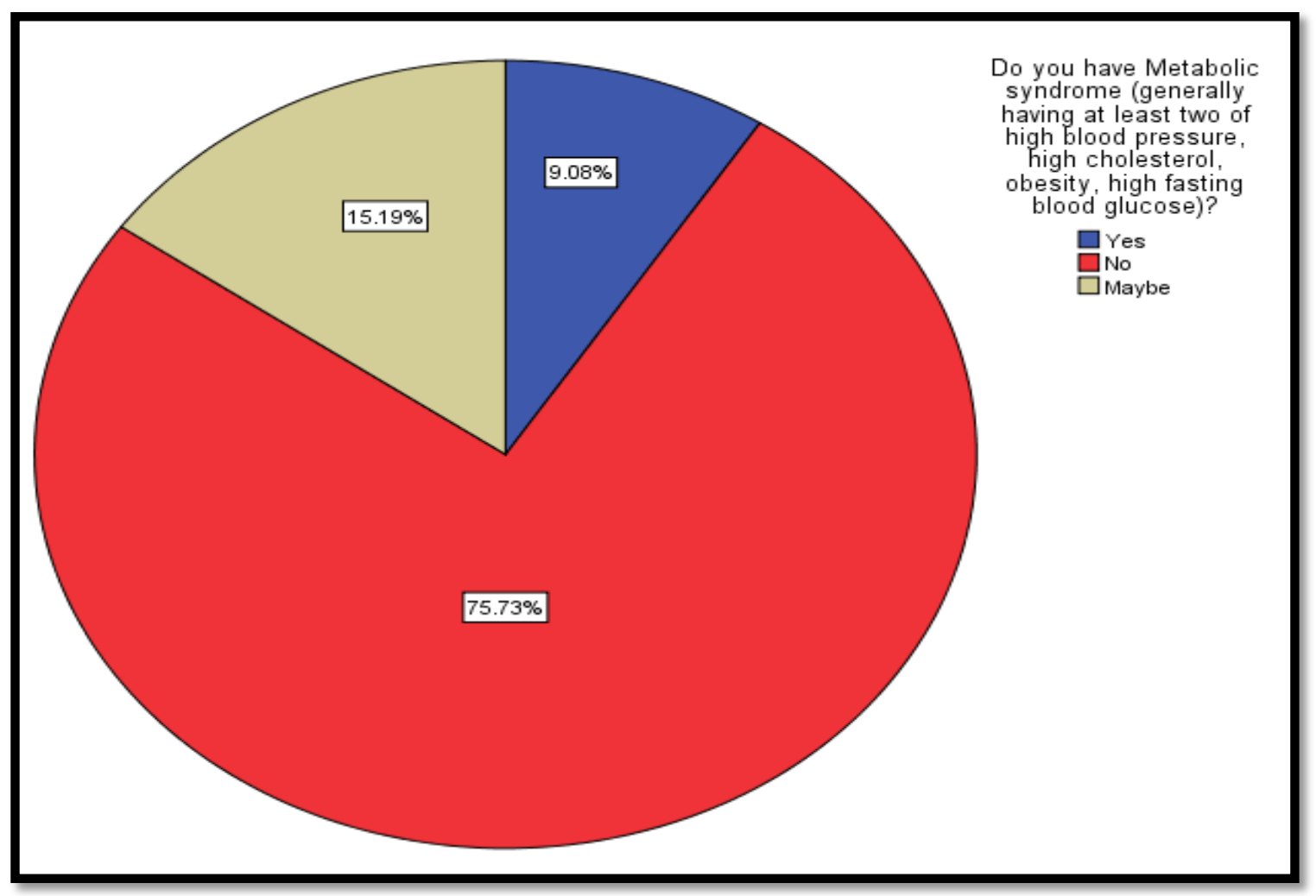

The results indicated that $75.73 \%$ of the respondents do not have Metabolic syndrome. 


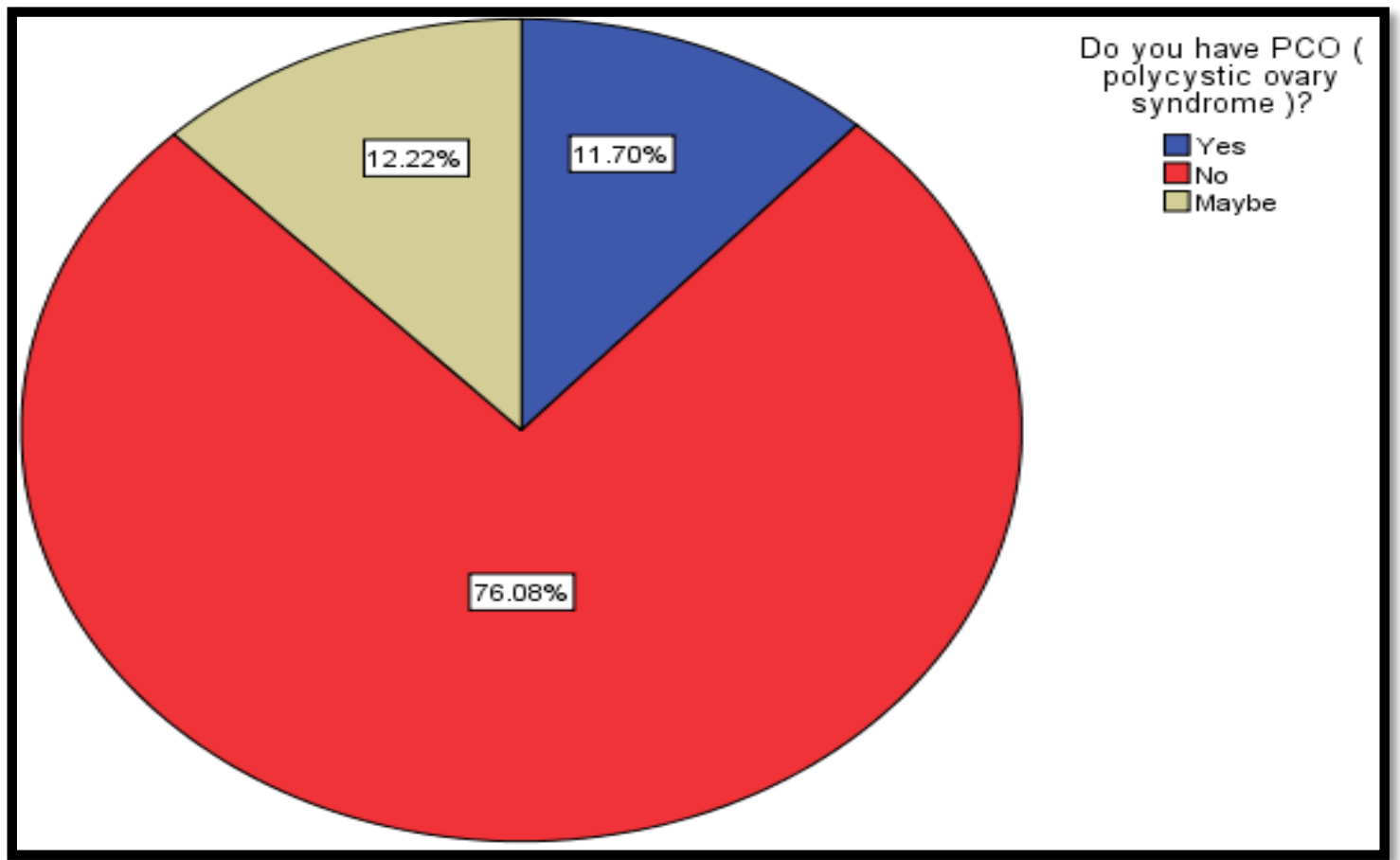

The results indicated that $76.08 \%$ of the respondents do not have $\mathrm{PCO}$ (polycystic ovary syndrome).

\begin{tabular}{|c|c|c|c|c|c|}
\hline \multicolumn{6}{|c|}{ Correlations } \\
\hline & Age & Nationality & Social status. & $\begin{array}{l}\text { Do You Have } \\
\text { Children? }\end{array}$ & $\begin{array}{c}\text { How old were } \\
\text { you at } \\
\text { puberty? }\end{array}$ \\
\hline Age & $\begin{array}{r}1 \\
-\end{array}$ & $\begin{array}{r}-.065- \\
.007\end{array}$ & $\begin{array}{c}.581 \\
.000\end{array}$ & $\begin{array}{r}-.654- \\
.000\end{array}$ & $\begin{array}{r}.026 \\
.273 \\
\end{array}$ \\
\hline & 1718 & 1718 & 1718 & 1718 & 1718 \\
\hline Nationality & $\begin{array}{r}-.065- \\
.007 \\
1718\end{array}$ & $\begin{array}{r}1 \\
1718\end{array}$ & $\begin{array}{r}-.041- \\
.088 \\
1718\end{array}$ & $\begin{array}{r}.080 \\
.001 \\
1718\end{array}$ & $\begin{array}{r}.022 \\
.369 \\
1718\end{array}$ \\
\hline Social_status & $\begin{array}{c}.581 \\
.000 \\
1718 \\
\end{array}$ & $\begin{array}{r}-.041- \\
.088 \\
1718 \\
\end{array}$ & $\begin{array}{r}1 \\
1718 \\
\end{array}$ & $\begin{array}{r}-.681- \\
.000 \\
1718 \\
\end{array}$ & $\begin{array}{r}.017 \\
.470 \\
1718 \\
\end{array}$ \\
\hline Do You Have Children? & $\begin{array}{r}-.654 \\
.000 \\
1718\end{array}$ & $\begin{array}{c}.080 \\
.001 \\
1718 \\
\end{array}$ & $\begin{array}{r}-.681- \\
.000 \\
1718\end{array}$ & $\begin{array}{r}1 \\
1718\end{array}$ & $\begin{array}{r}-.010- \\
.675 \\
1718 \\
\end{array}$ \\
\hline $\begin{array}{l}\text { How old were you at } \\
\text { puberty? }\end{array}$ & $\begin{array}{r}.026 \\
.273 \\
1718\end{array}$ & $\begin{array}{r}.022 \\
.369 \\
1718 \\
\end{array}$ & $\begin{array}{r}.017 \\
.470 \\
1718\end{array}$ & $\begin{array}{r}-.010- \\
.675 \\
1718\end{array}$ & $\begin{array}{r}1 \\
1718 \\
\end{array}$ \\
\hline Weight gain or obesity & $\begin{array}{r}-.247- \\
.000 \\
1718\end{array}$ & $\begin{array}{r}.029- \\
.229 \\
1718\end{array}$ & $\begin{array}{r}-160- \\
.000 \\
1718\end{array}$ & $\begin{array}{c}166 \\
.000 \\
1718\end{array}$ & $\begin{array}{c}.055 \\
.021 \\
1718\end{array}$ \\
\hline Pre-diabetes & $\begin{array}{r}-172- \\
.000 \\
1718\end{array}$ & $\begin{array}{r}.010- \\
.671 \\
1718 \\
\end{array}$ & $\begin{array}{r}-.074- \\
.002 \\
1718\end{array}$ & $\begin{array}{c}.078 \\
.001 \\
1718 \\
\end{array}$ & $\begin{array}{r}-.008- \\
.735 \\
1718\end{array}$ \\
\hline Type 2 diabetes & $\begin{array}{r}-119- \\
.000 \\
1718\end{array}$ & $\begin{array}{r}.010 \\
.686 \\
1718\end{array}$ & $\begin{array}{r}-.070- \\
.003 \\
1718\end{array}$ & $\begin{array}{r}.015 \\
.526 \\
1718\end{array}$ & $\begin{array}{r}.007 \\
.765 \\
1718\end{array}$ \\
\hline
\end{tabular}




\begin{tabular}{|c|c|c|c|c|}
\hline \multicolumn{5}{|c|}{ Correlations } \\
\hline & $\begin{array}{l}\text { Weight gain } \\
\text { or obesity }\end{array}$ & Pre-diabetes & $\begin{array}{c}\text { Type } 2 \\
\text { diabetes }\end{array}$ & $\begin{array}{l}\text { Cardiovascul } \\
\text { ar disease }\end{array}$ \\
\hline Age & $\begin{array}{r}-.247- \\
.000 \\
1718\end{array}$ & $\begin{array}{r}-.172- \\
-000 \\
1718\end{array}$ & $\begin{array}{r}-119- \\
-000 \\
1718\end{array}$ & $\begin{array}{r}-130- \\
-000 \\
1718\end{array}$ \\
\hline Nationality & $\begin{array}{r}-.029- \\
.229 \\
1718\end{array}$ & $\begin{array}{r}-.010- \\
-671 \\
1718\end{array}$ & $\begin{array}{r}-010 \\
-686 \\
1718\end{array}$ & $\begin{array}{r}-.046- \\
-054 \\
1718\end{array}$ \\
\hline Social_status & $\begin{array}{r}-160- \\
.000 \\
1718\end{array}$ & $\begin{array}{r}-074- \\
-002 \\
1718\end{array}$ & $\begin{array}{r}-070- \\
-003 \\
1718\end{array}$ & $\begin{array}{r}-085- \\
-000 \\
1718\end{array}$ \\
\hline Do You Have Children? & $\begin{array}{c}166 \\
.000 \\
1718\end{array}$ & $\begin{array}{c}.078 \\
.001 \\
1718\end{array}$ & $\begin{array}{r}-015 \\
-526 \\
1718\end{array}$ & $\begin{array}{r}-039 \\
-106 \\
1718\end{array}$ \\
\hline $\begin{array}{l}\text { How old were you at } \\
\text { puberty? }\end{array}$ & $\begin{array}{c}.055 \\
.021 \\
1718\end{array}$ & $\begin{array}{r}.008- \\
-735 \\
1718\end{array}$ & $\begin{array}{r}.007 \\
-765 \\
1718 \\
\end{array}$ & $\begin{array}{r}.002 \\
.927 \\
1718 \\
\end{array}$ \\
\hline Weight gain or obesity & $\begin{array}{r}1 \\
1718\end{array}$ & $\begin{array}{r}.133 \\
.000 \\
1718\end{array}$ & $\begin{array}{r}-014 \\
-565 \\
1718\end{array}$ & $\begin{array}{r}.059 \\
.014 \\
1718\end{array}$ \\
\hline Pre-diabetes & $\begin{array}{l}-133 \\
.000 \\
1718\end{array}$ & $\begin{array}{r}1 \\
1718\end{array}$ & $\begin{array}{r}-.014- \\
-568 \\
1718\end{array}$ & $\begin{array}{l}.061 \\
.011 \\
1718\end{array}$ \\
\hline Type 2 diabetes & $\begin{array}{r}.014 \\
.565 \\
1718\end{array}$ & $\begin{array}{r}-.014- \\
-568 \\
1718\end{array}$ & $\begin{array}{r}1 \\
1718\end{array}$ & $\begin{array}{r}.090 \\
.000 \\
1718\end{array}$ \\
\hline
\end{tabular}

**. Correlation is significant at the 0.01 level (2-tailed).

*. Correlation is significant at the 0.05 level (2-tailed).

\begin{tabular}{|c|c|c|c|}
\hline & \multicolumn{3}{|c|}{ Correlations } \\
\hline & $\begin{array}{c}\text { Endometrial } \\
\text { cancer }\end{array}$ & Sleep apnoea & $\begin{array}{c}\text { None of the } \\
\text { above }\end{array}$ \\
\hline Age & $\begin{array}{r}-.036 \\
.137 \\
1718\end{array}$ & $\begin{array}{r}.023 \\
.331 \\
1718\end{array}$ & $\begin{array}{c}-271 \\
.000 \\
1718\end{array}$ \\
\hline Nationality & $\begin{array}{r}-.069- \\
.004 \\
1718\end{array}$ & $\begin{array}{r}.021 \\
.374 \\
1718\end{array}$ & $\begin{array}{r}.029 \\
.235 \\
1718\end{array}$ \\
\hline Social_status & $\begin{array}{r}-.042- \\
.082 \\
1718\end{array}$ & $\begin{array}{r}.003 \\
.905 \\
1718\end{array}$ & $\begin{array}{r}-157 \\
.000 \\
1718\end{array}$ \\
\hline Do You Have Children? & $\begin{array}{r}-.017- \\
.486 \\
1718\end{array}$ & $\begin{array}{r}-.028- \\
.238 \\
1718\end{array}$ & $\begin{array}{r}.156- \\
.000 \\
1718\end{array}$ \\
\hline $\begin{array}{l}\text { How old were you at } \\
\text { puberty? }\end{array}$ & $\begin{array}{r}-.020- \\
.414 \\
1718\end{array}$ & $\begin{array}{r}.021 \\
.386 \\
1718\end{array}$ & $\begin{array}{r}-.046- \\
.055 \\
1718\end{array}$ \\
\hline Weight gain or obesity & $\begin{array}{r}.035 \\
.145 \\
1718\end{array}$ & $\begin{array}{r}.008 \\
.740 \\
1718\end{array}$ & $\begin{array}{r}-846- \\
.000 \\
1718\end{array}$ \\
\hline Pre-diabetes & $\begin{array}{r}-.009- \\
.723 \\
1718\end{array}$ & $\begin{array}{r}.003 \\
.899 \\
1718\end{array}$ & $\begin{array}{r}-.299- \\
.000 \\
1718\end{array}$ \\
\hline Type 2 diabetes & $\begin{array}{r}-172 \\
.000 \\
1718\end{array}$ & $\begin{array}{r}.015 \\
.537 \\
1718\end{array}$ & $\begin{array}{r}-.234- \\
.000 \\
1718\end{array}$ \\
\hline
\end{tabular}

**. Correlation is significant at the 0.01 level (2-tailed). 


\begin{tabular}{|c|c|c|}
\hline & & Zorrelations \\
\hline & 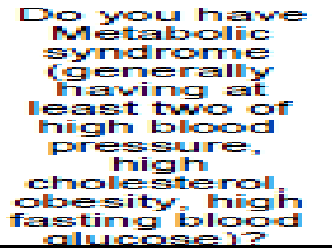 & 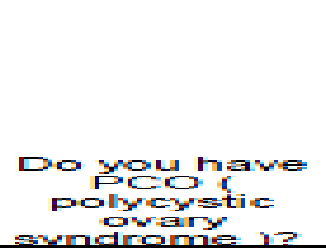 \\
\hline$\Delta 9=$ & $\begin{array}{r}-980 \\
-1700 \\
1710\end{array}$ & $\begin{array}{r}-1005 \\
1700 \\
170\end{array}$ \\
\hline Naticanzalits & $\begin{array}{r}-004- \\
-870 \\
17-18\end{array}$ & $\begin{array}{r}-00=- \\
-93= \\
17-10\end{array}$ \\
\hline Sociall_status & $\begin{array}{r}-047- \\
-040 \\
17-1=\end{array}$ & $\begin{array}{r}-03-1- \\
2 \geq 05 \\
17-13\end{array}$ \\
\hline 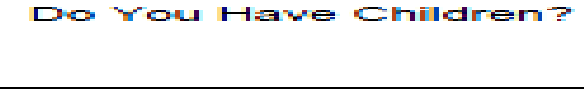 & $\begin{array}{r}-09= \\
-165 \\
-17-13\end{array}$ & $\begin{array}{r}-945 \\
-903 \\
17-10 \\
\end{array}$ \\
\hline Hubs ald were sou at & $\begin{array}{r}-0 \leq 4- \\
-\square \geq 5 \\
17-1: 3\end{array}$ & $\begin{array}{r}02-1 \\
31-1 \\
17-10\end{array}$ \\
\hline Weight gain or obesits & $\begin{array}{r}-013 \\
-580 \\
17+13\end{array}$ & $\begin{array}{r}070 \\
190= \\
17-13\end{array}$ \\
\hline$P r e-d i a b e t e s$ & $\begin{array}{r}-002 \\
-910 \\
17-10\end{array}$ & $\begin{array}{r}05-1 \\
1036 \\
17-10\end{array}$ \\
\hline Trpe 2 diabetes & $\begin{array}{r}-1 \geq 9 \\
-900 \\
-17-10\end{array}$ & $\begin{array}{r}002 \\
-9-1= \\
17-1=\end{array}$ \\
\hline
\end{tabular}

\begin{tabular}{|c|c|c|c|c|c|}
\hline \multicolumn{6}{|c|}{ Correlations } \\
\hline & Ace & Nationality & Social status & $\begin{array}{l}\text { Do You Have } \\
\text { children? }\end{array}$ & $\begin{array}{c}\text { How old were } \\
\text { you at } \\
\text { puberty? }\end{array}$ \\
\hline \multirow[t]{3}{*}{ Cardiovascular disease } & -.130 & $-.046-$ & -.085 & .039 & .002 \\
\hline & .000 & .054 & .000 & .106 & .927 \\
\hline & 1718 & 1718 & 1718 & 1718 & 1718 \\
\hline \multirow[t]{3}{*}{ Endometrial cancer } & $-.036-$ & -.069 & $-042-$ & $-.017-$ & -.020 \\
\hline & .137 & .004 & .082 & .486 & .414 \\
\hline & 1718 & 1718 & 1718 & 1718 & 1718 \\
\hline \multirow[t]{3}{*}{ Sleep apnoea } & .023 & .021 & .003 & $-.028-$ & .021 \\
\hline & .331 & .374 & .905 & .238 & .386 \\
\hline & 1718 & 1718 & 1718 & 1718 & 1718 \\
\hline \multirow[t]{3}{*}{ None of the above } & 271 & .029 & .157 & $-.156-$ & $-.046-$ \\
\hline & .000 & .235 & .000 & .000 & .055 \\
\hline & 1718 & 1718 & 1718 & 1718 & 1718 \\
\hline \multirow{3}{*}{$\begin{array}{l}\text { Do you have Metabolic } \\
\text { syndrome (generally } \\
\text { having at least two of high } \\
\text { blood pressure, high } \\
\text { cholesterol, obesity, high } \\
\text { fasting blood glucose)? }\end{array}$} & -.086 & $-.004-$ & $-.047-$ & .034 & $-.054-$ \\
\hline & .000 & .870 & .049 & .165 & .025 \\
\hline & 1718 & 1718 & 1718 & 1718 & 1718 \\
\hline \multirow{3}{*}{$\begin{array}{l}\text { Do you have PCO ( } \\
\text { polycystic ovary syndrome } \\
\text { )? }\end{array}$} & $-.005-$ & $-.002-$ & $-031-$ & .045 & .024 \\
\hline & .838 & .933 & .205 & .063 & .311 \\
\hline & 1718 & 1718 & 1718 & 1718 & 1718 \\
\hline
\end{tabular}




\begin{tabular}{|c|c|c|c|c|}
\hline \multicolumn{5}{|c|}{ Correlations } \\
\hline & $\begin{array}{l}\text { Weight gain } \\
\text { or obesity }\end{array}$ & Pre-diabetes & $\begin{array}{c}\text { Type } 2 \\
\text { diabetes }\end{array}$ & $\begin{array}{l}\text { Cardiovascul } \\
\text { ar disease }\end{array}$ \\
\hline \multirow[t]{3}{*}{ Cardiovascular disease } & .059 & .061 & .090 & 1 \\
\hline & .014 & .011 & .000 & \\
\hline & 1718 & 1718 & 1718 & 1718 \\
\hline \multirow[t]{3}{*}{ Endometrial cancer } & .035 & -.009 & .172 & .099 \\
\hline & .145 & .723 & .000 & .000 \\
\hline & 1718 & 1718 & 1718 & 1718 \\
\hline \multirow[t]{3}{*}{ Sleep apnoea } & .008 & .003 & .015 & .062 \\
\hline & .740 & .899 & .537 & .010 \\
\hline & 1718 & 1718 & 1718 & 1718 \\
\hline \multirow[t]{3}{*}{ None of the above } & -.846 & $-299-$ & $-234-$ & $-.198-$ \\
\hline & .000 & .000 & .000 & .000 \\
\hline & 1718 & 1718 & 1718 & 1718 \\
\hline \multirow{3}{*}{$\begin{array}{l}\text { Do you have Metabolic } \\
\text { syndrome (generally } \\
\text { having at least two of high } \\
\text { blood pressure, high } \\
\text { cholesterol, obesity, high } \\
\text { fasting blood glucose)? }\end{array}$} & .013 & & & .115 \\
\hline & .580 & .010 & .000 & .000 \\
\hline & & 1718 & 1718 & 1718 \\
\hline \multirow{3}{*}{$\begin{array}{l}\text { Do you have PCO ( } \\
\text { polycystic ovary syndrome } \\
\text { )? }\end{array}$} & .070 & .051 & .002 & .019 \\
\hline & .004 & .036 & .943 & -423 \\
\hline & 1718 & 1718 & 1718 & 1718 \\
\hline
\end{tabular}

\begin{tabular}{|c|c|c|}
\hline & \multicolumn{2}{|r|}{ Correlations } \\
\hline & 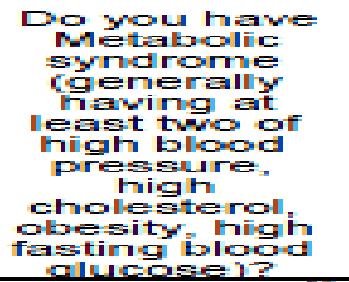 & 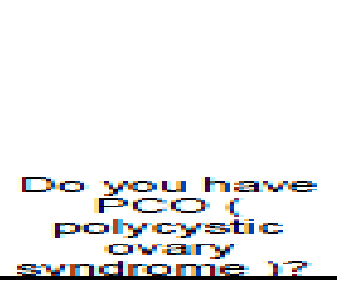 \\
\hline Cardiowascular disease & $\begin{array}{r}-115 \\
-1000 \\
17-10\end{array}$ & $\begin{array}{r}-019 \\
-120 \\
17-1: 3\end{array}$ \\
\hline Endometrial cancer & $\begin{array}{r}-062 \\
-010 \\
1719\end{array}$ & $\begin{array}{r}-057- \\
-019 \\
17-19\end{array}$ \\
\hline Slesep aprionea & $\begin{array}{r}-004 \\
-800 \\
17-10\end{array}$ & $\begin{array}{r}-1020 \\
-400 \\
17-10\end{array}$ \\
\hline Wone of the abowe & $\begin{array}{r}-104 \\
-153 \\
1710 \\
\end{array}$ & $\begin{array}{r}-061- \\
-01 \geq \\
1719\end{array}$ \\
\hline 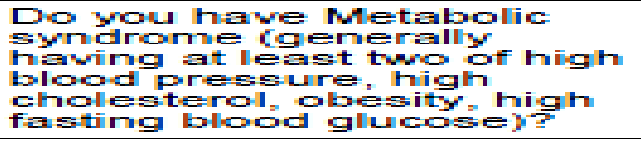 & $17+19$ & $\begin{array}{r}\cos 0 \\
-039 \\
17+1=\end{array}$ \\
\hline 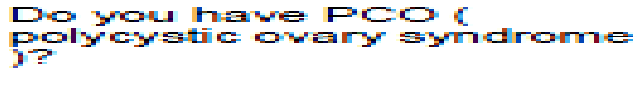 & $\begin{array}{l}050 \\
-0=9 \\
1710\end{array}$ & $+17: 3$ \\
\hline
\end{tabular}




\section{Discussion:-}

This study was based on a number of 1718 participants, 973 of them have children , 201 of them have PCO ( polycystic ovary syndrome ). which is considering as unhealthy sign .

The current study showed statistically significant ( $\mathrm{P}$ value is 0.004$)$ which means there is a strong relationship between having PCO and suffering from weight gain or obesity, ( $\mathrm{P}$ value is 0.036 ) which means there is a strong relationship between having $\mathrm{PCO}$ and suffering from pre-diabetes.

( $\mathrm{P}$ value is 0.019) which means there is a strong relationship between having PCO and suffering from Endometrial cancer, ( $\mathrm{P}$ value is 0.039 ) which means there is a strong relationship between having PCO and suffering from Metabolic syndrome.

\section{Conclusion:-}

The results of the study showed positive results in terms of the low incidence of PCO. There are some signs that may be associated with PCO, including weight gain and Pre-diabetes and they are considered as the most common causes related to PCO through the results. It has also been proven that there is a strong correlation between endometrial cancer and PCO infection, but the results have proven that a small number of women respondents have contracted the disease. There is also a strong correlation between metabolic syndrome and PCO infection, but the results demonstrate that few women respondents have the disease.

\section{Recommendation:-}

we recommend setting up health education programs about PCO, This health problem must be presented broadly and beneficially and, in a way, that everyone understands, as most deal with PCO by ignoring and not being important, work should be done on health conferences and medical discussions on that.

\section{Acknowledgement:-}

The authors would like to thank the participants for their great cooperation, Participants will be especially from medical universities and colleges selected and carried out by questionnaire.

\section{We thank the data collectors: \\ Ethical consideration:}

1. Individual consent from participants ( written on the front page of the questionnaire).

2. All information will be kept confidential.

\section{Budget:}

Self-funded.

\section{References:-}

1. Mayo Clinic. (2019). Polycystic ovary syndrome (PCOS) - Symptoms and causes. [online] Available at: https://www.mayoclinic.org/diseases-conditions/pcos/symptoms-causes/syc-20353439 [Accessed 24 Aug. 2019].

2. Jean Hailes For Women's Health. (2019). Polycystic ovary syndrome (PCOS). [online] Available at: https://jeanhailes.org.au/health-a-z/pcos/complications [Accessed 24 Aug. 2019].

3. N, A. (2017). Prevention of type 2 diabetes mellitus in polycystic ovary syndrome: A review. - PubMed - NCBI. [online] Ncbi.nlm.nih.gov. Available at: https://www.ncbi.nlm.nih.gov/pubmed/28711517 [Accessed 24 Aug. 2019].

4. Shaman, A., Mukhtar, H. and Mirghani, H. (2017). Risk factors associated with metabolic syndrome and cardiovascular disease among women with polycystic ovary syndrome in Tabuk, Saudi Arabia.. [online] PubMed. Available at: https://www.ncbi.nlm.nih.gov/pubmed/29403608 [Accessed 24 Nov. 2017].

5. Ezzidi I, e. (2018). Impact of variants on type-2 diabetes risk genes identified through genomewide association studies in polycystic ovary syndrome: a case-control study. - PubMed - NCBI. [online] Ncbi.nlm.nih.gov. Available at: https://www.ncbi.nlm.nih.gov/pubmed/30555071 [Accessed Dec. 2018].

6. Al-Nozha O, e. (2013). Body weight reduction and metformin: Roles in polycystic ovary syndrome. - PubMed NCBI. [online] Ncbi.nlm.nih.gov. Available at: https://www.ncbi.nlm.nih.gov/pubmed/23608322 [Accessed 20 Apr. 2013].

7. Al-Ruhaily, A., Malabu, U. and Malabu, R. (2019). Hirsutism in Saudi females of reproductive age: a hospital-based study.. [online] PubMed. Available at: https://www.ncbi.nlm.nih.gov/pubmed/18299651 [Accessed 31 Jan. 2008]. 